\title{
Long-Term Outcomes of Endoscopic Submucosal Dissection of Undifferentiated-Type Early Gastric Cancer
}

\author{
Chang Seok Bang ${ }^{1,2,3,4}$ \\ ${ }^{1}$ Department of Internal Medicine, Hallym University College of Medicine, Chuncheon, ${ }^{2}$ Institute for Liver and Digestive Diseases, \\ Hallym University, Chuncheon, ${ }^{3}$ Institute of New Frontier Research, Hallym University College of Medicine, Chuncheon, ${ }^{4}$ Division of Big \\ Data and Artificial Intelligence, Chuncheon Sacred Heart Hospital, Chuncheon, Korea
}

See "Endoscopic Submucosal Dissection versus Surgery for Undifferentiated-Type Early Gastric Cancer: A Systematic Review and Meta-Analysis" by Cheal-Wung Huh, Dae Won Ma, Byung-Wook Kim, et al., on page 202-210.

Endoscopic submucosal dissection (ESD) is the standard treatment for the subset of patients with early gastric cancer (EGC) satisfying prespecified criteria. The long-term prognosis following ESD for cases of EGC meeting the ESD criteria (achievement of curative resection) is comparable to that achieved with surgical resection. ${ }^{1}$ However, this long-term prognosis comparison categorized by the histology according to the differentiation, especially for undifferentiated-type EGC, has not been well established.

In the context of histology, undifferentiated-type EGC generally refers to poorly-differentiated tubular adenocarcinoma, signet-ring cell carcinoma, or poorly cohesive carcinoma. ${ }^{2}$ Although undifferentiated-type EGC is included among the expanded indications of ESD, the rate of curative resection remains low, reported previously as $61.4 \%$ in a meta-analysis and $36.4 \%$ in a nationwide cohort study in Korea. ${ }^{3,4}$ This implies that an unmet need persists regarding the accurate prediction of curative resection in undifferentiated-type EGC (difficulty in adopting a precise ESD indication). Therefore,

\footnotetext{
Received: January 7, 2021 Revised: January 16, 2021

Accepted: January 16, 2021

Correspondence: Chang Seok Bang

Department of Internal Medicine, Hallym University College of Medicine, 77 Sakju-ro, Chuncheon 24253, Korea

Tel: +82-33-240-5821, Fax: +82-33-241-8064, E-mail: csbang@hallym.ac.kr ORCID: https://orcid.org/0000-0003-4908-5431
}

(c) This is an Open Access article distributed under the terms of the Creative Commons Attribution Non-Commercial License (http://creativecommons.org/ licenses/by-nc/3.0) which permits unrestricted non-commercial use, distribution, and reproduction in any medium, provided the original work is properly cited. proper candidate selection prior to ESD is important.

Endoscopists predict the probability of curative resection using the size and shape of the lesion and whether ulcers are present or not. The most fundamental hypothesis is that endoscopic resection can be performed with curative intent for EGCs without lymph node metastasis. Therefore, indications of ESD were established using the combination of factors associated with a negligible lymph-node metastasis rate from the retrospective analysis of surgically resected specimens. ${ }^{2}$ These indications are categorized as per the histology of EGC according to the differentiation, size, and morphologic conditions of the involved lesion. However, optical endoscopic determination of the factors stated above involves operator-dependent characteristics. In the study of a Korean multicenter registry of ESD for undifferentiated-type EGC, there was a discrepancy between pre-ESD indications and post-ESD criteria in $36.7 \%$ of all lesions. ${ }^{4}$ The underestimation of the size was the most common reason for non-curative resection (71.4\%), followed by the underestimation of the invasion depth (32\%) and unpredictability of lymphovascular invasion (14.9\%). ${ }^{4}$ Undifferentiated-type EGC has distinctive growth patterns relative to differentiated-type EGC., ${ }^{2,4}$ Undifferentiated-type EGC is known to extend laterally along the proliferative zone in the intermediate layer of the mucosa (subepithelial spreading), and the development pattern from the intermediate layer could lead to non-exposure to the surface mucosa, limiting the precise measurement of the size of the lesion. ${ }^{3}$ Subepithelial-spreading signet-ring cell carcinoma is more prevalent than the epithelial-spreading type in cases with background 
gastric mucosal atrophy or intestinal metaplasia. Furthermore, ESD of the poorly-differentiated adenocarcinoma presents a stronger association with submucosal invasion relative to that of signet-ring cell carcinoma. ${ }^{4}$ Although adopting a precise indication is important, undifferentiated-type EGC itself is a risk factor for an out-of-indication rate, leading to non-curative resection, so more strict indications might be necessary for pursuing the ESD of undifferentiated-type EGC. ${ }^{5}$

In this issue of Clinical Endoscopy, Huh et al. reported a comparative evaluation of the long-term outcomes of ESD and surgery for undifferentiated-type EGC. ${ }^{6}$ There was no difference in the overall survival and procedure-related adverse events with the five Korean studies of 429 and 1,236 patients who underwent ESD and surgery, respectively. However, ESD showed a higher recurrence rate and lower complete resection rate compared to those of surgery. Although the overall survival was not different between patients who underwent ESD and surgery in this meta-analysis, this could change depending on how the ESD indication was applied in each enrolled study. Four studies (among the five studies included in the meta-analysis) included resected lesions based on the expanded indication and showed no significant difference in overall survival between ESD and surgery. However, there is difficulty in adopting a precise indication in the ESD of undifferentiated-type EGC. This means ESD is often performed on the basis of the beyond expanded indication which is mistaken for expanded indication. Therefore, there could be a selection bias in the results of this meta-analysis because patients with lesions for which the expanded indication was applied correctly (satisfying expanded criteria) were retrospectively enrolled in each study. If we could adopt a precise indication of ESD, the prognosis would be good. One of the methods to compensate this unmet need would be the application of strict indications (i.e., ESD for smaller lesions) because the underestimation of the size was the most common reason for non-curative resection in a previous study. ${ }^{4}$

Although a prospective trial of ESD for undifferentiated-type EGC that satisfied the expanded criteria reported an excellent long-term survival rate, ${ }^{4,7,8}$ more cautious application or restriction of ESD indications has been recommended, especially regarding the size categorization. ${ }^{2}$ Most recently published studies have also indicated that small intramucosal undifferentiated-type EGC measuring less than $1.0 \mathrm{~cm}$ or 1.5 $\mathrm{cm}$ without lymphovascular invasion should be considered for ESD. ${ }^{9,10}$

The indications of ESD for undifferentiated-type EGC have not been approved by all endoscopists. Therefore, practice patterns adopting ESD indications for undifferentiated-type EGC vary depending on the institution. However, the results of previous studies commonly suggested that ESD for the lesions satisfying the expanded criteria has a good prognosis. Therefore, efforts to apply precise indications are important.

\section{Conflicts of Interest}

Chang Seok Bang has been an Associate Editor member of Clinical Endoscopy; however, he was not involved in the peer reviewer selection, evaluation, or decision process of this article. The author has no potential conflicts of interest.

\section{Funding}

Funding for this research was provided by the Bio \& Medical Technology Development Program of the National Research Foundation (NRF), the Korean government, Ministry of Science and ICT (MSIT) (grant number NRF2017M3A9E8033253), and the Korean College of Helicobacter and Upper Gastrointestinal Research Foundation Grant (2017-08).

\section{Author Contributions}

Writing-original draft: Chang Seok Bang

Writing-review\&editing: CSB

ORCID

Chang Seok Bang:

https://orcid.org/0000-0003-4908-5431

\section{REFERENCES}

1. Jeon HK, Kim GH, Lee BE, et al. Long-term outcome of endoscopic submucosal dissection is comparable to that of surgery for early gastric cancer: a propensity-matched analysis. Gastric Cancer 2018;21:133-143.

2. Bang CS, Baik GH. Pitfalls in the interpretation of publications about endoscopic submucosal dissection of early gastric cancer with undifferentiated-type histology. Clin Endosc 2019;52:30-35.

3. Bang CS, Baik GH, Shin IS, et al. Endoscopic submucosal dissection for early gastric cancer with undifferentiated-type histology: a meta-analysis. World J Gastroenterol 2015;21:6032-6043.

4. Bang CS, Park JM, Baik GH, et al. Therapeutic outcomes of endoscopic resection of early gastric cancer with undifferentiated-type histology: a Korean ESD registry database analysis. Clin Endosc 2017;50:569-577.

5. Horiuchi Y, Fujisaki J, Yamamoto N, et al. Undifferentiated-type component mixed with differentiated-type early gastric cancer is a significant risk factor for endoscopic non-curative resection. Dig Endosc 2018;30:624-632.

6. Huh CW, Ma DW, Kim BW, Kim JS, Lee SJ. Endoscopic submucosal dissection versus surgery for undifferentiated-type early gastric cancer: a systematic review and meta-analysis. Clin Endosc 2021;54:202-210.

7. Jeon HK, Lee SJ, Kim GH, Park DY, Lee BE, Song GA. Endoscopic submucosal dissection for undifferentiated-type early gastric cancer: shortand long-term outcomes. Surg Endosc 2018;32:1963-1970.

8. Lee S, Choi KD, Han M, et al. Long-term outcomes of endoscopic submucosal dissection versus surgery in early gastric cancer meeting expanded indication including undifferentiated-type tumors: a criteria-based analysis. Gastric Cancer 2018;21:490-499.

9. Liang XQ, Wang Z, Li HT, et al. Indication for endoscopic treatment based on the risk of lymph node metastasis in patients with undifferentiated early gastric cancer. Asian J Surg 2020;43:973-977.

10. Ryu DG, Choi CW, Kim SJ, et al. Possible indication of endoscopic resection in undifferentiated early gastric cancer. Sci Rep 2019;9:16869. 